\title{
A Research on the Electrical Test Fault Diagnostic and Data Mining of a Manned Spacecraft
}

\author{
Feng Yang ${ }^{1,2}, \mathrm{Ye} \mathrm{Lv}^{3,2}$, and Wenting $\mathrm{Li}^{4}$ \\ ${ }^{1}$ Department of Aeronautics and Astronautics Engineering, Nanjing University of Aeronautics and Astronautics, Nanjing, \\ 210016, China \\ ${ }^{2}$ Institute of Manned Space System Engineering, China Academy of Space Technology, Beijing, 100094, China \\ ${ }^{3}$ School of information system and management, National University of Defense Technology, Changsha, 410073, China \\ ${ }^{4}$ Beijing Aerospace Automatic Control Institute, Beijing, 100039, China
}

\begin{abstract}
The paper introduces the modeling method and modeling tool for the fault diagnosis of manned spacecraft, the multi-signal flow graph model of a manned space equipment was established using this method; the framework of the fault detection and diagnosis system of manned spacecraft is proposed, the function of ground system and function of the spacecraft are clearly defined. The structure of the functional module is given separately; finally, the tool builds the fault detection and diagnosis system, the application of fault diagnosis method for manned spacecraft is used for reference.
\end{abstract}

\section{INTRODUCTION}

Manned spacecraft is a large complex system with the latest cutting-edge achievements in the field of machinery, electronics, materials, control, energy, communication and computer technology. Because of the design level, manufacturing technology, the influence of such factors as cost limits, the manned spacecraft reliability of each subsystem, component is reduced, and thus prone to failure, affect the flight mission, threatened the personal safety, and manned spacecraft to reliable operation in the task of thousands years' time. Therefore, the design of manned spacecraft must be capable of fault monitoring and diagnosis, which is an extremely important auxiliary means to improve the reliability of manned spacecraft [1 3].

In recent years, the research on spacecraft fault diagnosis method has been paid more and more attention. Various fault diagnosis methods have advantages and disadvantages, and the fault diagnosis of spacecraft is a complicated problem. In general, it is not only expected to be able to diagnose faults in individual subsystems and components, but also to diagnose at the system level. By comparing various fault diagnosis methods, it can be seen that each method has its own advantages and disadvantages, and different research objects apply different diagnostic methods. Based on the analysis of the subjects and the advantages and disadvantages of various diagnostic methods, this paper selects the model based method to study the fault diagnosis of manned spacecraft. [4 6].

\section{Fault Monitoring and Diagnosis Key Technology Research}

The model-based fault monitoring and diagnosis method mainly involves the modelling, fault monitoring and diagnosis system architecture, monitoring and diagnosis system construction and other key technologies.

\subsection{Modelling Methods and Tools}

Currently in use within the territory of the fault signal modelling methods mainly include relational database modelling method, the information flow model modelling method and the model of multiple signal flow graph modelling method, through the comparison, this paper select multiple signal flow graph model modelling method.

\subsubsection{Model Modelling Method}

Conceptually, the multi-signal flow graph model covers multiple information flow models, and the model is closer to the physical structure of the system. In addition, "signal" corresponds to the independent unit in the transmission function of the system or the unique attribute that constitutes the function of the system [3]. Because the model is very similar to the real system structure, the model integration and verification are greatly simplified and effectively improve the efficiency of the model builders.

Formally speaking, the multi-signal flow graph model consists of the following elements:

- A finite system constitutes a set of components, $C=\left\{c_{1}, c_{2}, \cdots, c_{L}\right\}$;

- System - related independent signal sets $V=\left\{v_{1}, v_{2}, \cdots, v_{k}\right\}$;

- $\mathrm{N}$ - dimensional test $\operatorname{set} T=\left\{t_{1}, t_{2}, \cdots, t_{n}\right\}$;

- $n_{p}$ dimensions can be used to test point sets $T P=\left\{T P_{1}, T P_{2}, \cdots, T P_{n_{p}}\right\}$

* Corresponding author: yangfeng501@,163.com 
- Each test point $T P_{p}$ corresponds to a set of tests $S P\left(T P_{P}\right)$;

- Each element $c_{i}$ affects a set of signals $S C\left(c_{i}\right)$;

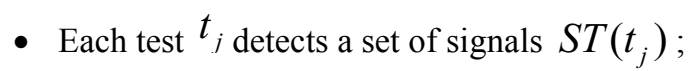

Directed graph $D G=\{C, T P, E\}$, there are the physical connections to the diagram to represent the system.

The multi-signal flow graph model has advantages in obtaining the important knowledge of system fault diagnosis, and the multi-signal flow graph modelling method does not require the system component to specify the failure mode knowledge. In the modelling process of multi-signal flow graph, the failure mode influence analysis is carried out through the failure mode description of the relevant signals of each part. The multi-signal flow graph modelling method represents the fault space of the system. Therefore, in the multi-signal flow graph method, only the propagation process of system component failure mode is modelled. Compared with quantitative modelling and qualitative modelling methods, the cost and difficulty of modelling are greatly reduced.

\subsubsection{Modelling Tools}

Modelling method has been supported by software tools, the TEAMS of both companies in the United States is a set of software testability, maintainability and system status monitoring function of the commercial software products, complex object can be static, dynamic testability analysis, mainly for defence and aviation, space systems, to provide users with advanced, highquality health diagnosis, prediction and system management products and solutions. At the same time, the real-time status monitoring and remote fault diagnosis of complex system can be realized through the supporting modules of TEAMS-RDS and TEAMS-RT.

The TEAMS-RT software is a supporting tool for online and real-time diagnosis. It is based on the team test model of the established system, as well as the $0 / 1$ state of each test point in the model (passed/not passed). Such a team -RT software can identify known good, bad, and suspect components.

The TEAMS-RT is designed for remote monitoring/diagnosis. Using this technique, users can upload sensors or test data to the RDS using the Internet, and they can be diagnosed in a few seconds by intelligent automatic processing based on the test case design model.

TEAMS software for testability analysis and evaluation of complex product, the real-time condition monitoring and remote diagnosis provides a good solution, can be on the basis of the test model to build real-time monitoring and diagnosis system of running status of manned spacecraft, and improves the automation of manned spacecraft, management level and management level.

\subsubsection{Modelling Steps}

During the study, follow these steps.

The first step, input structure model, schematic diagram model or concept block diagram. In the TEAMS, the structural model can be automatically generated or entered directly through the graphical user interface through VHDL structure model, EDIF wire table, etc.

Second, load the signal to the module and test point. A signal set can be found from a functional or pass function independent variable. For example, the signal characteristics of the power amplifier will include output distortion, harmonic distortion and power output. Generally any unique attribute has a relevant signal. For example, a bus with multiple independent addressable devices is used as a signal for each device's address.

Step 3: modify the model under special circumstances. When the following special situations occur in the modelling process (not necessarily all of them), the necessary corrections can be made. In the TEAMS, all of these fixes are available.

- If system with redundant structure composition (Both A and B have to be invalidated), redundant components to make use of the AND node set.

- If the system has a different mode of operation, the use of modeling the SWITCH node.

- If the system can contain parts in the test machine, the TEAMS should indicate in the modeling. It is particularly noteworthy that inmachine self-testing can be modeled with special types of signal names.

- If the system has a replaceable digital integrated circuit components, can be modeled by their equivalent model. The equivalent model is to simplify the chip model and obtain the necessary correlation information to detect faults in the chip (without fault isolation).

- In special circumstances, the system has a counter the correlation between each other, must identify and eliminate such correlation.

Step 4: Test the Model. The multi-signal model is tested according to the test data and physical model.

\subsection{Fault Monitoring and Diagnosis Technology of Manned Spacecraft}

\subsubsection{Overall Structure and Plan}

The manned spacecraft is based on the system structure of the fault monitoring and control system of the earth and earth. The fault monitoring and diagnosis system of world integration is mainly composed of monitoring and diagnosis system, ground monitoring and diagnosis system. On monitoring and diagnosis system is responsible for the spacecraft status monitoring, fault diagnosis and emergency treatment, the ground 
monitoring and diagnosis system is responsible for fault prediction, fault diagnosis and system reconfiguration, troubleshooting and manned spacecraft health status evaluation. The system architecture of fault monitoring and diagnosis system is shown in Fig. 1.

Among them on monitoring and diagnosis system has fault management architecture of data acquisition, data processing, condition monitoring (abnormal monitoring), health assessment, fault diagnosis and prediction) and decision support (fault disposal), and other functions.

The display and alarm system has status monitoring, health assessment support and decision support function. The display and warning system can simultaneously receive data from the monitoring and diagnosis system and ground monitoring and diagnostic system status monitoring results data, health assessment module output fault diagnosis data. In addition, the health assessment support and decision support module also provides an area of expertise in human-computer interaction.

Ground monitoring and diagnosis system for data processing, condition monitoring, health assessment, health, forecast and decision support function, under the support of experts in the field, with more accurate ability of health assessment and decision support.

Based on the existing state monitoring and diagnosis technology, the real-time status monitoring and fault location of manned spacecraft is realized based on the telemetry data and remote control instructions of manned spacecraft. Using Teams testability analysis software system model is set up, and to evaluate the testability of manned spacecraft, the output correlation matrix and diagnosis strategy, monitoring and diagnosis system based on Teams - RT on real-time condition monitoring and diagnosis, the ground to realize fault detection and diagnosis system combined with domain experts' further reasoning to locate the fault. The overall scheme of fault monitoring and diagnosis system for the integration of heaven and earth is shown in Fig.2.

\subsubsection{On Board Monitoring and Diagnosis System}

Shown on the monitor and diagnosis system structure in Fig. 4. IN figure 3 upper part, for the development of manned spacecraft system real-time monitoring and diagnosis system, firstly according to the need of multi signal flow graph modelling method mentioned in the introduction, the establishment of multi signal flow model of the diagnosed object using the TEAMS software, and then based on the interface file generation and RT TEAMS model, the interface file in the TEAMSDesigner environment can directly generate the suffix were INP, PRM, MAP file, and then according to the characteristics of manned spacecraft system of real-time fault diagnosis reasoning program. In order to facilitate the development of real-time fault diagnosis system, QSI provides a real time diagnostic reasoning application program interface -- TEAMS-RT API. In TEAMS-RT API, the input and output data format, the function code, and the returned diagnostic data format are provided for the interaction with the TEAMS dynamic link library.
Among them, the core code of real-time diagnosis reasoning is encapsulated in TEAMS dynamic link library. Real time diagnosis is the use of TEAMS-RT API development for the application of the system, the real-time file regularly read the test results and the model, called TEAMS.dll for diagnostic reasoning and the diagnosis result output process.

\subsubsection{Ground monitoring and diagnosis system}

He structure of the ground monitoring and diagnosis system is shown in Fig. 3, below half part, the ground monitoring and diagnosis system based on Browser / server $(\mathrm{B} / \mathrm{S})$ architecture, the server real-time diagnostic procedures and database management system, the user can receive authorization in computer network browsing real-time monitoring results.

The ground monitoring and diagnosis system is mainly composed of three parts: ground control system, diagnosis centre server and remote diagnostic resource (expert). The ground control system receives manned spacecraft telemetry parameters, send control commands, receive the telemetry parameters; diagnosis centre server is the core logic of diagnosis system, it mainly includes the data processing module, monitoring module, fault diagnosis module, remote expert consultation platform and based on Web database and knowledge base system, provides intelligent diagnosis and maintenance support service for the manned spacecraft; remote diagnosis resources (expert) is to provide the technical support of remote real-time diagnosis system of manned spacecraft, solve the difficult fault.

The ground monitoring and diagnosis system of manned spacecraft system has the following processing steps:

- Data acquisition: manned spacecraft operation data management system for acquisition, processing and storage of manned spacecraft operation data, the data is formatted in the packaging process, the channel encoding and subcarrier modulation after telemetry downlink RF telemetry channel is sent to the center.

- Comprehensive diagnosis: telemetry center sends data to the diagnostic server for processing after being readable. The diagnostic center server in real-time monitoring and fault reasoning module of manned spacecraft system into a multi signal model based on the parameters obtained on the basis of inference, can quickly isolate the fault, and according to the prior knowledge base generating maintenance suggestions. Manned spacecraft system complex if the automatic fault isolation tools cannot real-time situation, immediately set up interactive diagnosis task and expert system, the expert system in the browser can access to Web diagnosis center server, access to the existing state of manned spacecraft, and according to the information of the remote expert consultation.

- Maintenance suggestions: system fault isolation, fault maintenance suggestions generated through the browser interface, feedback to the transportation management center of manned 
spacecraft guidance and technical personnel, repair tasks.

\section{Intelligent mining technology of test data}

In spacecraft testing, data interpretation is an important part. At present, the main data interpretation system is based on the division system, and the main interpretation methods are:

- Draw the curve of the parameter test data to the time, and judge whether the jump point is reasonable.

- The criterion interval of the correct value of the given parameter, and the transfinite data is determined to be abnormal.

The main disadvantages of current interpretation methods are:

- Unable to interpret the change trend of parameters in a large period of time. Using the current interpretation method, the interpretation interval is concentrated on a certain day or a test stage, and is not compared with the previous stage, the previous model test data, cannot see the changing trend of data.

- It is impossible to interpret the possible differences in the status of multiple similar products. The spacecraft has multiple telemetry parameters of the same or similar function, which characterize the states of the same kind of products. The current interpretation method does not focus on the specific interpretation of such parameters, and cannot find the differences between similar products.

- Neglecting the connection between parameters. The current interpretation method uses single parameter as the unit to interpret the data, ignoring the specific relationship between some parameters. Using the current interpretation method, easy to cause the following situation, lead to leakage judgment: in a number of correlation parameters, the test data of each parameter are in the normal range, but the parameters do not conform to the curve.

In view of the above advantages and disadvantages analysis, we have carried on the data intelligent mining analysis research. Data intelligent mining analysis refers to the automatic data query and processing through the database, to achieve the same parameter test data longitudinal ratio, the same parameter test data transverse ratio, correlation parameter test data joint ratio.

Users can query the historical test data of multiple spacecraft by software, and then pre-process the data and compare the data. The work flow is shown in figure 4. There are two ways to deal with data: image processing and statistical processing. Image processing is to display one or more test data visually on the image, the horizontal axis can be time, data serial number, can also be a data. Statistical processing refers to the selection of different parameters in a certain period of time, to facilitate users to find outliers.

\section{Conclusions}

This paper studied by monitoring and fault diagnosis method of manned spacecraft, a test model of a typical aerospace equipment, construction of the monitoring and fault diagnosis system of the system, realize real-time monitoring, remote client access and interactive expert diagnosis, provides a reference scheme of monitoring and fault diagnosis of manned spacecraft. The results show that:

- Through the research of system level testability design method, master the multi signal flow chart modeling method and modeling tools, and provide reference for the system level testability design of spacecraft;

- The thought of fault monitoring and diagnosis system is put forward, the construction monitoring and diagnosis system for spacecraft integrated, and ground monitoring and fault location reasoning of the spacecraft fault at the same time, the two complement each other, the thought is of great significance for the space station and other large spacecraft fault diagnosis;

- The real-time algorithm of spacecraft fault monitoring and diagnosis is introduced, and the real-time diagnosis algorithm is optimized according to the characteristics of manned spacecraft, and the optimized algorithm flow is given;

Finally, according to the method studied in this paper, using the "three ratio" test data intelligent mining method, the test data is further analyzed and verified.

\section{References}

1. Y. Yan, H.X. Wu, X.L. Wang, Z.B. Li. Overview of Spacecraft Fault Diagnosis and Fault Tolerant Control Technology. Journal of Astronautics, 2013, 24(3): 221-226.

2. B. Long. The Research of Multi-signal Flow Modelling and Fault Diagnosis Method used in Spacecraft. Harbin Industry Technology, 2005: Harbin.

3. J.Y. Shi, X. Zhang, T.G. Zhou. Application of Multi-signal Modelling and Diagnosis Strategy Design Technology. Systems Engineering and Electronics, 2011, 33(4):811-815, 2011.

4. R. Sakthivel, S. Selvi, K. Mathiyalagan Fault Tolerant Sampled Data Control of Flexible Spacecraft with Probabilistic Time Delays. Nonlinear Dynamics, 2014, 79(3):1835-1846.

5. Y. Shen, X.L. Zhang, Z.H. Wang. Spacecraft Fault Diagnosis Based on Empirical Mode Decomposition and Directed Factor Graph. Journal of Harbin Institute of Technology, 2013, 45(1):19-24.

6. H. Kato, J. Ota. Real-time Spacecraft Actuator Fault Diagnosis with State Segmented Particle Filtering. Advanced Robotics, 2014, 28(19): 1265-1276. 


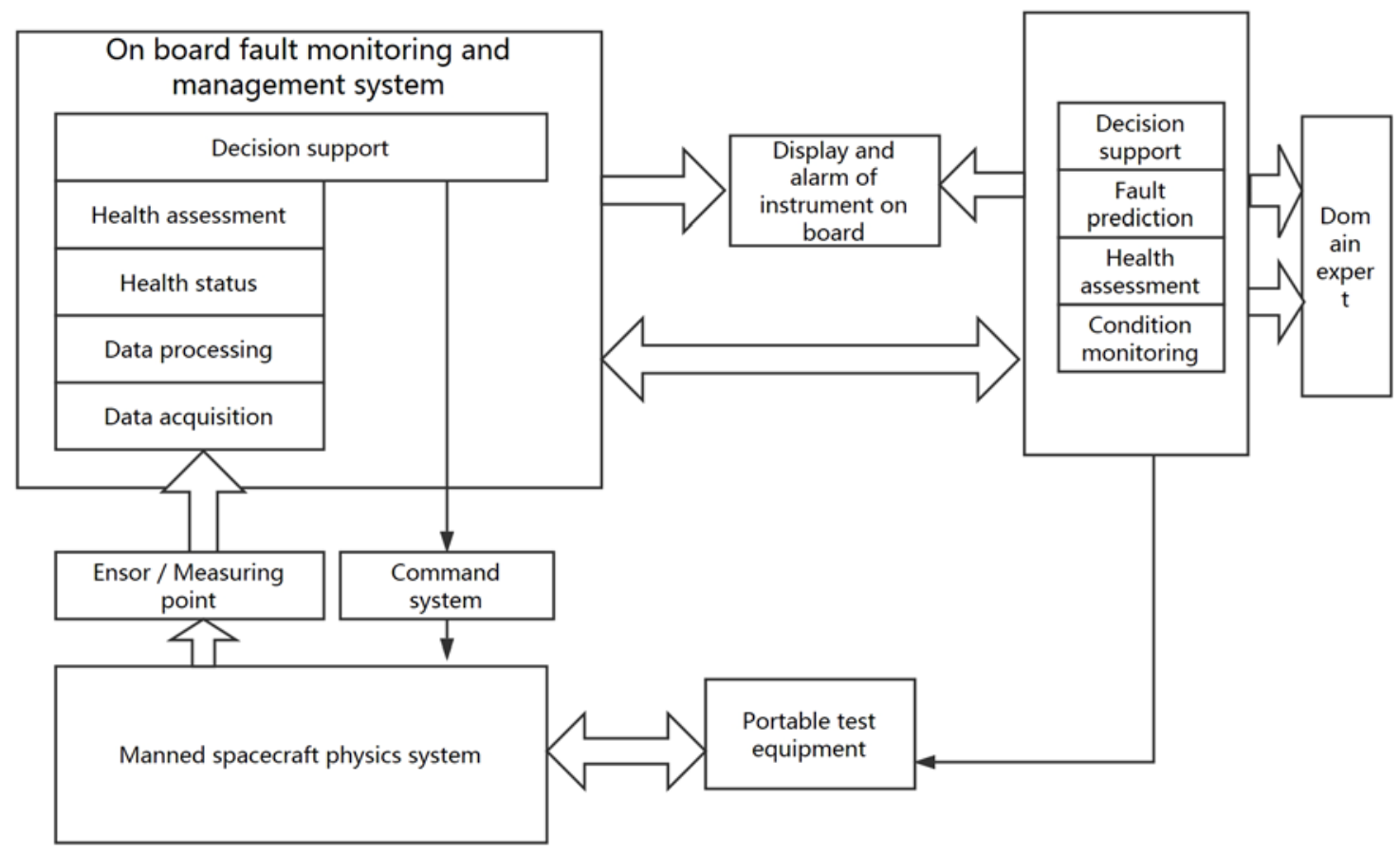

Fig 1. Fault monitoring and diagnosis system architecture based on integration of earth and sky

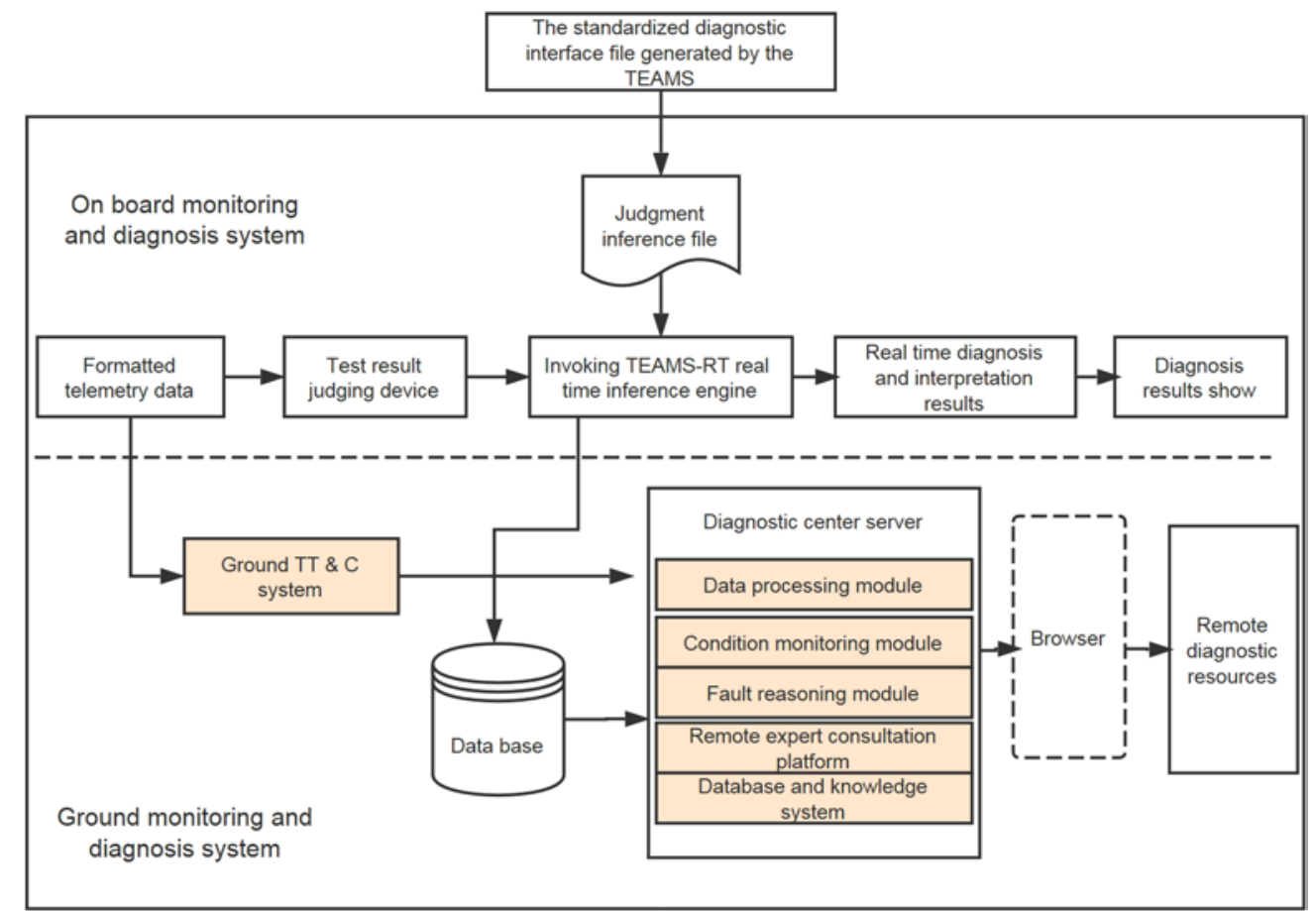

Fig 2. Overall scheme of fault monitoring and diagnosis for integration of heaven and earth 